\title{
Erratum
}

\section{Anesthésie locale en chirurgie buccale chez les sujets cardiaques}

\author{
Fadila Daïmellah ${ }^{1, \star}$, Saléha Lehachi ${ }^{1}$, Zakia Bennoui ${ }^{1}$, Saïda Khelil ${ }^{1}$, Mohand-Saïd Issad ${ }^{1}$, \\ Hamid Boukais ${ }^{2}$, Wacila Zerrouki ${ }^{2}$, Mohamed Berkane ${ }^{2}$, Djohar Hannoun ${ }^{3}$, \\ Samira Abrouk ${ }^{3}$ \\ 1 Service de Cardiologie, CHU Béni Messous, Alger, Algérie \\ 2 Service de Pathologie et Chirurgie buccales, CHU Béni Messous, Alger, Algérie \\ 3 Institut national de Santé publique (INSP) Alger, Algérie
}

(Rectificatif demandé par les auteurs de l'article, Med Buccale Chir Buccale 2010;16:79-84, D0I: 10.1051/mbcb/2010011)

Dans le chapitre Matériel et méthodes, le paragraphe Participants débute par la phrase suivante : « Cette étude a été effectuée parallèlement avec une autre étude dont l'objectif principal était de comparer l'incidence du saignement après des extractions dentaires chez des patients sous acénocuoumarol aux doses thérapeutiques versus le relais par l'héparine calcique » [2].

Cette formulation est ambiguë et elle peut laisser penser que l'on a effectué deux études différentes. L'objectif de la première étant de comparer l'incidence du saignement après extractions dentaires chez deux groupes de patient, les uns sous acénocoumarol, les autres sous relais héparinique, l'objectif de la deuxième étant d'identifier les évènements cardiovasculaires indésirables survenant au décours d'extractions dentaires réalisées sous mépivacaine avec noradrénaline chez des patients atteints de diverses maladies cardiovasculaires.

En fait, il s'agit d'une seule étude qui avait un objectif principal et plusieurs objectifs secondaires. L'objectif principal était de comparer l'incidence du saignement après extractions dentaires chez des patients sous acénocoumarol ou sous relais héparinique. Un des objectifs secondaires de cette même étude consistait à identifier les évènements cardiovasculaires indésirables survenant au décours d'extractions réalisées avec un anesthésique contenant un vasoconstricteur ; dans cette étude, toutes les extractions dentaires ont été effectuées mépivacaine avec noradrénaline.

Par conséquent, pour être plus précis, on aurait dû dire en parlant des participants à l'étude : « Cet essai thérapeutique concernait des patients traités par les antivitamines $\mathrm{K}$ au long cours. Il avait pour principal objectif de comparer l'incidence du saignement après des extractions dentaires réalisées en poursuivant le traitement par acénocoumarol aux doses thérapeutiques ou en effectuant un relais avec l'héparine calcique [2]. Tous les malades étaient hospitalisés dans le service de Cardiologie pour diverses pathologies cardiovasculaires et nécessitaient des extractions dentaires simples ou chirurgicales, uniques ou multiples, en une ou plusieurs séances. Ont été exclus de l'étude les patients présentant une HTA non stabilisée (pression artérielle lors du bilan initial supérieure ou égale à $160-95 \mathrm{~mm} \mathrm{Hg}$ ), un IDM de moins de 3 semaines, un angor non stabilisé par le traitement ou de troubles de la conduction à l’ECG nécessitant l'implantation d'un pacemaker ».

^Correspondance : daimfadila@yahoo.fr 